\title{
The Association between Stress Level in Daily Life and Age at Natural Menopause in Korean Women: Outcomes of the Korean National Health and Nutrition Examination Survey in 2010-2012
}

Byoung-O Choi, Yeon-Ji Lee*, Ji-Ho Choi, Se-Wook Cho, Hyun-Jung Im, Jee-Eun An

Department of Family Medicine, Inha University School of Medicine, Incheon, Korea

Background: Although several risk factors associated with reduced age at natural menopause (ANM) have been investigated, the results are inconsistent. Excessive stress, which leads to elevation of stress hormones, can also negatively affect reproductive ability, including by accelerating menopause. However, a direct association between stress level and ANM has not yet been demonstrated. Therefore, the object of this study was to investigate the association between stress level and ANM in Korean women.

Methods: Study participants were Korean women between 40 and 70 years old who were in natural menopause during the 5th Korean National Health and Nutrition Examination Survey $(n=3,176)$. The level of stress in daily life was estimated based on data from the mental health topics of the survey. We used the $t$-test and one-way analysis of variance to analyze the correlation between stress level and ANM. Regression ( $\beta$ ) coefficients calculated by multiple regression analysis were used to estimate various factors affecting ANM.

Results: Women who experienced a high level of stress in daily life had a lower mean ANM than women with a low stress level (50.17 \pm 3.7 and $50.58 \pm 3.5$ years, respectively), with a statistically significant correlation $(\mathrm{P}<0.05)$. This correlation was still observed after adjusting for age, body mass index, menstrual regularity, and personal income $(\mathrm{P}<0.05$ for variables).

Conclusion: In Korean women between 40 and 70 years of age who are in natural menopause, there is a statistically significant correlation between stress level and ANM. In particular, women who experience a high level of stress in daily life have reduced ANM.

Keywords: Menopause; Stress 


\section{INTRODUCTION}

Natural menopause refers to the natural permanent cessation of reproductive ability and menstrual cycle due to the decline of the ovarian function without secondary causes such as surgery, chemotherapy, administration of drugs, and diseases. Age at natural menopause is affected by genetic factors related to region and ethnicity and various environmental factors such as socioeconomic position, life style, and culture. ${ }^{1-3)}$ Postmenopausal women are constantly exposed to the risk of chronic diseases due to the reduction in the level of ovarian hormones such as estrogen and suffer from corresponding symptoms. ${ }^{4-6)}$ Therefore, age at natural menopause (ANM) is an important risk factor of morbidity and mortality, and women who had early menopause are more likely to develop osteoporosis, ${ }^{7)}$ obesity, metabolic syndrome, ${ }^{8-10)}$ and cardiovascular diseases. ${ }^{11-14)}$ Previous studies revealed that income level, occupation, marital status, age at menarche, parity, smoking, and stress might affect reproductive ability and age at menopause, but their results were inconsistent. ${ }^{15-17)}$

Stress can be defined as the response of the body to internal and external threat factors and the consequences of this response. Stress exerts physiological effects on peripheral organs, starting from the central nervous system, via hormonal secretion. ${ }^{18)}$ The purpose of this process is to properly adapt to the changes in the surrounding environment. Although transient stress may accelerate adaptation, chronic stress may cause a variety of mental and physical problems. Therefore, stress has recently drawn attention as a cause and exacerbating factor of various diseases. Excessive stress in daily life is known to cause diabetes, hypertension, hyperlipidemia, cardiovascular diseases, cognitive disorders, depression, digestive diseases, and chronic fatigue. Hyperactive hypothalamic-pituitary-adrenal axis as activated by stress, and elevated levels of stress hormones such as glucocorticoids and catecholamines are thought to affect the development and progression of such diseases. ${ }^{19)}$ Moreover, elevated levels of stress hormones can cause gynecological diseases such as amenorrhea or early menopause. Nevertheless, a direct association between stress and ANM has not yet been demonstrated in Korea or abroad.

Therefore, we analyzed the association between level of stress in daily life and ANM in Korean women based on the data from the 5th Korean Health and Nutrition Examination Survey.

\section{METHODS}

\section{Study Subjects}

Out of 13,918 women participating in the 5th Korean Health and Nutrition Examination Survey (2010-2012), 3,176 adult women between 40 and 70 years old who had natural menopause were selected for this study. In order to rule out second- ary causes that might affect age of menopause, women with premature menopause (before 40 years old, $n=29$ ), those who underwent oophorectomy $(n=17)$, those with a history of hormone therapy $(\mathrm{n}=402)$, and those with a history of oral contraceptives use $(n=742)$ were excluded from the present study. Women who did not complete the survey or had missing anthropometric values $(n=45)$ were also excluded. Finally, a total of 1,941 women were included in the present study.

\section{Survey and Measurement Methods}

As in the previous surveys, in the 5th Health and Nutrition Examination Survey (2010-2012), a health questionnaire and health examination survey were conducted in mobile health screening vehicles, and interviews and a self-administered questionnaire were used to assess major disease management and morbidity. Direct measurements, observations, and sample analyses were used, and height and weight were measured with accuracies of $\pm 0.1 \mathrm{~cm}$ and $\pm 0.1 \mathrm{~kg}$, respectively. Body mass index (BMI) was calculated using the formula (weight $[\mathrm{kg}] /$ square of height $\left[\mathrm{m}^{2}\right]$ ). Stress level in daily life was surveyed using four grades: (1) "I am extremely stressed," (2) "I am very stressed," (3) "I am moderately stressed," and (4) "I feel little stress." For stress evaluation, items 1 and 2 were classified as high level of stress while items 3 and 4 were classified as low level of stress.

\section{Definition of Menopause}

Although appropriate biological markers for the diagnosis of menopause are not available, it can be diagnosed when the level of follicle-stimulating hormone is increased to at least 50 $\mathrm{IU} / \mathrm{L}$, the serum estradiol concentration is reduced to $50 \mathrm{pg} /$ $\mathrm{mL}$ or less, and the woman has not had a spontaneous menstrual period for 1 year or more. ${ }^{20)}$ However, these diagnostic criteria were not utilized in the Korean Health and Nutrition Examination Survey, with menopause detected based on selfreported amenorrhea and natural menopause.

\section{Statistics}

For analysis of basal characteristics of the study subjects, values were expressed as mean and standard deviation or ratio (\%). The independent sample t-test and one-way analysis of variance (one-way ANOVA) were used to detect correlations between independent stress-related variables and ANM. In order to adjust for confounders that can affect ANM, multiple regression analysis was performed for age, BMI, menstrual cycle regularity, and personal income. All statistical analyses were conducted using IBM SPSS ver. 19.0 (IBM Co., Armonk, NY, USA). The significance level was set at $\mathrm{P}<0.05$.

\section{RESULTS}

The basal characteristics of the subjects are presented in Table 
Table 1. Basal characteristics of the study subjects from the Korean National Health and Nutrition Examination Survey $(n=1,941)$

\begin{tabular}{lc}
\hline Characteristic & Value \\
\hline Age $(\mathrm{y})$ & $59.33 \pm 6.3$ \\
$40-49$ & $64(3.3)$ \\
$50-59$ & $954(49.1)$ \\
$60-70$ & $923(47.6)$ \\
Body mass index $\left(\mathrm{kg} / \mathrm{m}^{2}\right)$ & $24.30 \pm 3.3$ \\
High $(>25)$ & $749(38.6)$ \\
Normal (18.5-25) & $1,157(59.6)$ \\
Low $(<18.5)$ & $35(1.8)$ \\
Stress recognition level & \\
Severe to extreme & $477(24.6)$ \\
Mild to moderate & $1,464(75.4)$ \\
Menstrual cycle regularity & $1,768(91.1)$ \\
Experience of live births & $1,889(97.3)$ \\
Ever smoker & $100(5.2)$ \\
Personal income & \\
High & $482(24.8)$ \\
Upper middle & $494(25.5)$ \\
Lower middle & $469(24.2)$ \\
Low & $496(25.6)$
\end{tabular}

Values are presented as mean \pm standard deviation or number (\%).

Table 3. Changes in age at natural menopause according to stress recognition level by multiple regression analysis

\begin{tabular}{lcc}
\hline Variable & $\beta$ (stand.) & P-value $^{*}$ \\
\hline Model 1 & -0.053 & 0.020 \\
Model 2 & -0.053 & 0.020 \\
Model 3 & -0.055 & 0.014 \\
Model 4 & -0.050 & 0.023 \\
\hline
\end{tabular}

Model 1 was adjusted for age. Model 2 was adjusted for age and BMI. Model 3 was adjusted for age, BMl, and menstrual cycle regularity. Model 4 was adjusted for age, $\mathrm{BMI}$, menstrual cycle regularity, and personal income.

BMl, body mass index.

${ }^{*}$ Obtained by multiple regression analysis.

1. The mean age $(n=1,941)$ was $59.33 \pm 6.3$ years, and the mean BMI was $24.3 \pm 3$.3. The number of women who reported a high level of stress in daily life was 477 (24.6\%), whereas the number of women who felt relatively little stress was 1,464 (75.4\%). The number of women with regular menstrual cycle was $1,768(91.1 \%)$, and the number of women with live birth(s) was 1,889 (97.3\%). The number of smokers was 100 (5.2\%). Personal income was expressed in quartiles (high, upper middle, lower middle, and low). Mean values of basal characteristics that might affect age at menopause are compared in Table 2. To compare mean levels of stress in daily life and ANM, the subjects were divided into 3 subgroups by age (40-49, 50-59, and $60-70$ years). Although no statistically significant differences were found between the subgroups, all of them showed a persistent tendency of a higher stress level being linked to a lower ANM. A comparison with mean overall values revealed that a high level of stress in daily life was significantly associated with an ANM reduced by about 5 months ( $50.58 \pm 3.5$ years vs. $50.17 \pm 3.7$ years, $\mathrm{P}<0.05)$. BMI and menstrual cycle regularity significantly affected ANM $(\mathrm{P}<$ 0.05 in both cases), whereas personal income, history of live
Table 2. Mean ANM according to stress, age, BMI, menstrual cycle regularity, history of live births, smoking, and personal income

\begin{tabular}{|c|c|c|}
\hline Variable & Value & P-value* \\
\hline \multicolumn{3}{|l|}{ Stress recognition level (y) } \\
\hline $40-49$ & & 0.509 \\
\hline Severe to extreme & $46.12 \pm 1.7$ & \\
\hline Mild to moderate & $46.51 \pm 2.1$ & \\
\hline $50-59$ & & 0.358 \\
\hline Severe to extreme & $50.55 \pm 2.6$ & \\
\hline Mild to moderate & $50.74 \pm 2.7$ & \\
\hline $60-70$ & & 0.071 \\
\hline Severe to extreme & $50.12 \pm 4.3$ & \\
\hline Mild to moderate & $50.68 \pm 4.1$ & \\
\hline Overall & & 0.029 \\
\hline Severe to extreme & $50.17 \pm 3.7$ & \\
\hline Mild to moderate & $50.58 \pm 3.5$ & \\
\hline $\mathrm{BMI}\left(\mathrm{kg} / \mathrm{m}^{2}\right)$ & & 0.017 \\
\hline High (>25) & $50.75 \pm 3.7$ & \\
\hline Normal (18.5-25) & $50.33 \pm 3.5$ & \\
\hline Low $(<18.5)$ & $49.66 \pm 3.4$ & \\
\hline Regular menstrual cycle & & 0.007 \\
\hline Yes & $50.41 \pm 3.6$ & \\
\hline No & $51.18 \pm 3.6$ & \\
\hline History of live births & & 0.084 \\
\hline Yes & $50.50 \pm 3.6$ & \\
\hline No & $49.63 \pm 3.4$ & \\
\hline Ever smoker & & 0.144 \\
\hline Yes & $49.97 \pm 3.7$ & \\
\hline No & $50.51 \pm 3.6$ & \\
\hline Personal income & & 0.091 \\
\hline High & $50.63 \pm 3.4$ & \\
\hline Upper middle & $50.70 \pm 3.3$ & \\
\hline Lower middle & $50.39 \pm 3.7$ & \\
\hline Low & $50.18 \pm 3.9$ & \\
\hline
\end{tabular}

Values are presented as mean ANM \pm standard deviation.

ANM, age at natural menopause; BMl, body mass index.

*Obtained by one-way analysis of variance or Student t-test.

births, and history of smoking had no effect. Women with lower BMI and more regular menstrual cycle were found to have earlier ANM. Table 3 shows changes in ANM after adjustment for each confounder. In model 1, only age was adjusted for, and a statistically significant correlation was detected $(\mathrm{P}<0.05)$. In models 2,3 , and 4 with additional adjustments for BMI, menstrual cycle regularity, and personal income level, respectively, statistically significant negative correlations were identified $(\mathrm{P}<0.05)$.

\section{DISCUSSION}

The present study demonstrated a significant association between level of stress in daily life and ANM. In addition, ANM was further reduced by low BMI in the presence of a high level of stress. Menopause is a time of very significant biological and social changes in a woman's life. Post-menopausal women may be constantly exposed to the risk of chronic disease. Although menopausal symptoms may occur during perimenopause, they are usually severe immediately after the onset of 
menopause. Post-menopausal women begin to feel a variety of mental and physical changes. Therefore, in the modern society where physical as well as perceived age becomes important, menopause is considered to be directly linked to the woman's quality of life. In the present study, a high level of stress was found to reduce the mean age at menopause by about 5 months from $50.58 \pm 3.5$ years to $50.17 \pm 3.7$ years $(\mathrm{P}<0.05)$. These results may be clinically significant because women with menopausal symptoms may visit an outpatient department and undergo examination to determine underlying diseases and receive treatment. As the mean age of women and their social engagement are increasing, studies investigating the variety of causes that can affect age at menopause become increasingly significant. Stress, one of these causes, may affect woman's reproductive ability, menstrual cycle, and ANM by altering the neuroendocrine system. However, despite a number of studies, the exact mechanism by which stress causes disease still remains uncertain. Allostatis is a good example of a relatively powerful model. In the late 1980s, Sterling and Eyer $^{21)}$ introduced allostatis to describe physiological changes resulting from the adaptation process and aimed at maintaining the internal stability under stress conditions. It was found that, if daily stimulation is repeated for a long period of time or the level of stimulation is very high, allostatic overload can occur in the process of adaptation even though homeostasis is maintained, and this may result in health problems through a variety of mechanisms. ${ }^{21}$ )

It has to be noted that, because in this cross-sectional study stress evaluation was limited to a single question ("What is your level of stress in daily life?"), it may be somewhat difficult to interpret the results. ${ }^{19)}$ Thus, although high body weight and smoking have been found to be major causes of decreased ANM in a number of previous studies, we did not detect such associations. ${ }^{22,23)}$ A possible explanation is that only presence of smoking was considered in the present study, without quantitative and temporal segmentation. Nevertheless, the existence of a direct association between stress and ANM in Korean women is an important finding. Therefore, the results of the present study may be used as a reference for similar future studies.

In summary, a high level of stress was found to be significantly associated with earlier natural menopause in Korean women between 40 and 70 years of age. This association was still present after adjusting for possible confounders.

\section{CONFLICT OF INTEREST}

No potential conflict of interest relevant to this article was reported.

\section{REFERENCES}

1. Thomas F, Renaud F, Benefice E, de Meeus T, Guegan JF. International variability of ages at menarche and menopause: patterns and main determinants. Hum Biol 2001;73:271-90.

2. Morabia A, Costanza MC. International variability in ages at menarche, first livebirth, and menopause. World Health Organization Collaborative Study of Neoplasia and Steroid Contraceptives. Am J Epidemiol 1998;148:1195-205.

3. Murabito JM, Yang Q, Fox C, Wilson PW, Cupples LA. Heritability of age at natural menopause in the Framingham Heart Study. J Clin Endocrinol Metab 2005;90:3427-30.

4. Research on the menopause in the 1990s: report of a WHO Scientific Group. World Health Organ Tech Rep Ser 1996;866:1-107.

5. Wilson MM. Menopause. Clin Geriatr Med 2003;19:483-506.

6. Cui R, Iso H, Toyoshima H, Date C, Yamamoto A, Kikuchi S, et al. Relationships of age at menarche and menopause, and reproductive year with mortality from cardiovascular disease in Japanese postmenopausal women: the JACC study. J Epidemiol 2006;16:177-84.

7. Kritz-Silverstein D, Barrett-Connor E. Early menopause, number of reproductive years, and bone mineral density in postmenopausal women. Am J Public Health 1993;83:983-8.

8. Gordon T, Kannel WB, Hjortland MC, McNamara PM. Menopause and coronary heart disease. The Framingham Study. Ann Intern Med 1978;89:157-61.

9. Jacobsen BK, Heuch I, Kvale G. Age at natural menopause and stroke mortality: cohort study with 3561 stroke deaths during 37-year followup. Stroke 2004;35:1548-51.

10. Sternfeld B, Bhat AK, Wang H, Sharp T, Quesenberry CP Jr. Menopause, physical activity, and body composition/fat distribution in midlife women. Med Sci Sports Exerc 2005;37:1195-202.

11. Snowdon DA, Kane RL, Beeson WL, Burke GL, Sprafka JM, Potter J, et al. Is early natural menopause a biologic marker of health and aging? Am J Public Health 1989;79:709-14.

12. Colditz GA, Stampfer MJ, Willett WC, Stason WB, Rosner B, Hennekens $\mathrm{CH}$, et al. Reproducibility and validity of self-reported menopausal status in a prospective cohort study. Am J Epidemiol 1987;126:319-25.

13. Cooper GS, Sandler DP. Age at natural menopause and mortality. Ann Epidemiol 1998;8:229-35.

14. Kelsey JL, Gammon MD, John EM. Reproductive factors and breast cancer. Epidemiol Rev 1993;15:36-47.

15. Van Noord PA, Dubas JS, Dorland M, Boersma H, te Velde E. Age at natural menopause in a population-based screening cohort: the role of menarche, fecundity, and lifestyle factors. Fertil Steril 1997;68:95102.

16. Hardy R, Kuh D, Wadsworth M. Smoking, body mass index, socioeconomic status and the menopausal transition in a British national cohort. Int J Epidemiol 2000;29:845-51.

17. Parazzini F; Progetto Menopausa Italia Study Group. Determinants of age at menopause in women attending menopause clinics in Italy. Maturitas 2007;56:280-7.

18. The Korean Academy of Family Medicine. Textbook of family medicine. 4th ed. Seoul: The Korean Academy of Family Medicine; 2013.

19. Shin HC. Measuring stress with questionnaires. J Korean Med Assoc 2013;56:485-95.

20. Hargrove JT, Eisenberg E. Menopause. Med Clin North Am 1995;79: 1337-56.

21. Sterling P, Eyer J. Allostasis: a new paradigm to explain arousal pathol- 
ogy. In: Fisher S, Reason J, editors. Handbook of life stress, cognition and health. New York (NY): John Wiley \& Sons; 1988. p. 629-49.

22. Pawlinska-Chmara R, Szwed A. How the age at menopause is related to cigarette smoking in Polish women? Acta Medica Lituanica 2005;12:
43-7.

23. Mikkelsen TF, Graff-Iversen S, Sundby J, Bjertness E. Early menopause, association with tobacco smoking, coffee consumption and other lifestyle factors: a cross-sectional study. BMC Public Health 2007;7:149. 